\title{
Pasado y presente de la renovación pedagógica en España (de finales del Siglo XIX a nuestros días). Un recorrido a través de escuelas emblemáticas
}

\section{Past and present of the pedagogic renewal (from the late nineteenth century to the present day) A tour through flagship schools}

\author{
Francisco Javier PERICACHO GÓMEZ \\ Universidad Complutense de Madrid
}

Recibido: Septiembre 2012

Aceptado: Noviembre 2012

\section{Resumen}

España alberga una rica y heterogénea historia de renovación pedagógica llena de iniciativas escolares que significaron una ruptura e innovación en cada momento histórico. En coherencia, a lo largo del presente artículo se examina su desarrollo desde finales del Siglo XIX hasta la actualidad, a través de un recorrido por diferentes centros escolares de educación primaria que han sido referentes y emblemáticos de la misma, provenientes tanto del ámbito público como privado. Se presenta la investigación desde una perspectiva cronológica que permite entender de forma gradual su desarrollo histórico y actual, en orden a generar una visión de conjunto que sintetiza pasado y presente.

La investigación se ha realizado examinando diferentes estudios sobre renovación pedagógica en España, prensa de la época y las principales revistas educativas que sirvieron de órganos de expresión en cada momento histórico. Se completa el trabajo incluyendo datos obtenidos mediante la revisión de proyectos educativos, la observación directa de algunas experiencias y la consulta a miembros relevantes de las mismas.

Palabras clave: renovación pedagógica en España, escuela, historia de la educación, escuela alternativa.

\footnotetext{
Abstract

Spain has a rich and varied history of pedagogical renewal full of school initiatives that meant a shift and an innovation in each historical period. Accordingly, this article examines its development since the late nineteenth century to the present day, throughout different private and public primary schools that have been emblematic and a reference. Consequently, this research is presented from a chronological perspective that gradually permits to understand its historical and current development, in order to generate an overview that summarizes the past and re-contextualizes the present.

This research has been carried out by examining different pedagogical renewal research in Spain, and different newspapers and magazines of the period, which served as organs of 
expression in each historical period. The work is completed with data obtained from the revision of educational projects, the direct observation of some experiences, and the consultation with relevant participants. .

Keywords: pedagogic renovation in Spain, school, history of education, alternative school.

El modelo de escuela actual sugiere un modo particular de organización y una forma institucional determinada, pero éste no es el único modelo posible de escuela, la única organización que puede calificarse como escolar ${ }^{1}$. Desde sensibilidades pedagógicas diferentes, a lo largo de la historia siempre han existido escuelas que han mostrado una mirada diferente sobre el sentido de la educación ${ }^{2}$. Su estudio histórico revela el inagotable surgimiento de iniciativas escolares que rompieron con la lógica pedagógica establecida. Experiencias que, desde una re-invención curricular integral, pretendieron ofrecer una respuesta diferente al modelo de escuela tradicional que Frabboni $^{3}$ caracteriza en base a tres puntos:

- Estructura: centralista, burocrática, jerárquica y separada del ambiente social. Aislamiento docente y centralidad didáctica del aula.

- Contenidos culturales: currículo fundado en la información y la transmisión de un saber dado, inactual y unilateral. Creatividad centrada en el lenguaje gráfico/figurativo.

- Didáctica: preponderancia del programa y la lección con protagonismo absoluto del docente. Gran importancia otorgada al manual y el ejercicio individual. Estructura rígida de horarios

Las prácticas de renovación pedagógica han representado (antes y ahora) una manera diferente de abordar los procesos educativos y de entender la institución escolar, una actitud que ha promovido la innovación constante con el fin de mejorar la respuesta que ofrecía dicha institución a los retos educativos que la sociedad planteaba ${ }^{4}$. En coherencia, partiendo de la necesidad de buscar incesantemente mayor calidad $^{5}$ y adecuación de la respuesta educativa a la realidad social, la renovación pedagógica es un concepto amplio y flexible que ha estado siempre íntimamente ligada al contexto histórico, político y social. En conclusión, es un conjunto organizado de actividades teóricas y prácticas, encaminadas a superar la realidad pedagógica dominante en un momento dado, ${ }^{6}$ representando una actitud crítica constante que a lo

\footnotetext{
${ }^{1}$ Viñao, A. (1990). Innovación pedagógica y racionalidad científica. La escuela graduada pública en España (1898-1936). Madrid: Akal

${ }^{2}$ Mateu, J. (2011). Circunstancias, retos y posibilidades de las escuelas alternativas. Organización y Gestión Educativa, 4, 17-20.

${ }^{3}$ Frabboni, F. (1998). El libro de la pedagogía y la didáctica: II.- Lugares y tiempos de la educación. Madrid: Editorial Popular.

${ }^{4}$ ITE (Instituto Nacional de Tecnologías Educativas y de Formación del Profesorado). La renovación pedagógica en el Estado Español. http://www.ite.educacion.es/ (Consultado el 5 de Enero de 2012).

${ }^{5}$ Domènech, J. (1995). Aproximació a la renovació pedagógica. Temps d'Educació, 14, 175- 184.

${ }^{6}$ Caivano, F. y Carbonell, J. (1979). La Renovación Pedagógica ayer y hoy. Cuadernos de Pedagogía, 59, s.p.
} 
largo de la historia se muestra favorable a la mejora de los procesos educativos y la institución escolar en general.

En España, desde finales del Siglo XIX y hasta nuestros días, esta actitud permanente de crítica, mejora e innovación de la escuela, ha generado un rico legado de iniciativas ${ }^{7}$ que se han alejado total o parcialmente del modelo escolar tradicional, constituyendo un modelo escolar alternativo ${ }^{8}$. En líneas generales, las señas de identidad quedan resumidas en tres aspiraciones pedagógicas con efecto en la totalidad de actores, escenarios y estructuras involucradas: consecución de una escuela activa en su metodología, democrática en sus estructuras y abierta en su relación con el medio. Un número considerable de experiencias que se imponen como cuestión e interrogante socio-pedagógico urgente y de entidad.

En conclusión, el objetivo de la presente investigación es estudiar diacrónicamente la renovación pedagógica desarrollada en España desde finales del Siglo XIX hasta la actualidad, a través de un recorrido por algunos de los centros escolares de educación primaria más emblemáticos (tanto públicos como privados). Centros que, en la mayor parte de los casos, sirvieron de referente e inspiración a otros. A tal fin, se presenta la investigación desde una perspectiva cronológica a través de la cual se van exponiendo las experiencias e identificando sus idearios pedagógicos. En este sentido, se comienza el itinerario pedagógico con la aparición de la emblemática Institución Libre de Enseñanza (ILE) en 1876 y se finaliza con iniciativas vigentes como la red de colegios "Amara Berri".

Este recorrido se ha elaborado a través del estudio de la producción científica sobre la renovación pedagógica desarrollada en España y la revisión, tanto de diferentes periódicos de la época como de las principales revistas que sirvieron de órganos de expresión de la misma en cada momento histórico. Asimismo, en cuanto a iniciativas vigentes en la actualidad, se completa la información incluyendo datos obtenidos mediante la observación directa de algunas experiencias y la consulta a miembros relevantes y proyectos educativos.

\footnotetext{
${ }^{7}$ Experiencias que han estado fuertemente influidas por las concepciones pedagógicas del movimiento de la "Escuela Nueva" que se inicia a finales del Siglo XIX. En este sentido, encontramos en Luzuriaga una precisa descripción y caracterización pedagógica (a partir de Ferrière) de las "Escuelas Nuevas" resumida en tres puntos: Educación Física (vida en el campo, agua, aire y luz en abundancia, equilibrio y salud del cuerpo considerado como la condición primordial de la salud del espíritu), Educación intelectual (ni memorización, ni erudición impuesta al niño de fuera adentro, reflexión y razón ejerciéndose de dentro a fuera, partir del hecho para elevarse a la idea, práctica del método científico) y Educación moral (educación para la iniciativa y la responsabilidad, la emancipación de la autoridad se hace por mérito personal, negativa al ejercicio de la autoridad ejercida de fuera adentro). Luzuriaga, L. (1923a). Las escuelas nuevas. Boletín de la Institución Libre de Enseñanza, tomo XLVII, 765, 304-309.

${ }^{8}$ Caracterización general del modelo escolar alternativo: estructura (descentralizada, experimental, horizontal, democrática y abierta al ambiente), contenidos culturales (el currículo integra la información, la investigación y la creatividad; ambiente utilizado con fines didácticos; fomento de la creatividad a través de múltiples lenguajes: gráfico/figurativo, mímico/gestual, manipulador, constructivo, musical, lúdico...) y didáctica (estructura de horarios flexible, trabajo en equipo, creación de grupos de estudio e investigación, uso de diferentes materiales y contextos de aprendizaje: manual, biblioteca, mediateca...). Síntesis realizada en base a Frabboni, F., op. Cit., 1998.
} 
Desde diferentes autores con estudios cercanos se apoya la presente investigación 9 . El merito e innovación que alberga la misma, reside en el esfuerzo por ofrecer un recorrido a través de las escuelas más emblemáticas, estableciendo una línea de continuidad que sintetiza y rastrea las huellas de la renovación pedagógica llegando a la actualidad. Se pretende, tanto continuar una línea de trabajo que estudia la renovación pedagógica interrogándose sobre su re-contextualización actual y recorrido histórico como servir de soporte y estímulo a futuras investigaciones.

Por último, se constata una realidad, este estudio es consciente de sus limitaciones de espacio, por ende, se hace responsable del amplio número de experiencias históricas y actuales que se no se cubren, en este sentido se aclara que únicamente se visibilizan algunas de las más reveladoras y emblemáticas. Asimismo, debido a las características de la historia de la renovación pedagógica de España y al pretendido esfuerzo de síntesis, la investigación se centra principalmente, aunque no exclusivamente, en las zonas geográficas de Madrid y Cataluña hasta 1975, por ser donde más posibilidades de desarrollo se dieron.

\section{Finales del Siglo XIX y comienzos del Siglo XX}

A finales del Siglo XIX la escuela española experimentaba un considerable atraso material y pedagógico en comparación con el resto de países europeos industrializados: profesores poco retribuidos, escasez de escuelas para cubrir la totalidad de población en edad escolar, desconsideración social y laboral del maestro, prácticas pedagógicas autoritarias y memorísticas, falta de democratización escolar... Las ideas de modernidad pedagógica y cambio sobre los métodos, los contenidos, el concepto de la infancia y la relación con la naturaleza que venían recorriendo España durante el Siglo XIX, unido a diferentes sensibilidades políticas y/o religiosas, logra cristalizar en la aparición de diferentes experiencias. Estos intentos de renovación educativa estuvieron motivados principalmente desde la iniciativa privada. Se pueden establecer tres corrientes básicas ${ }^{10}$.

1. Corriente de carácter laico-burgués, con Francisco Giner de los Ríos como figura esencial y la emblemática "Institución Libre de Enseñanza".

9 Caivano, F. y Carbonell, J., op. cit., 1979, s.p.; Viñes, C. (1983). La Renovación Pedagógica del Siglo XIX y las colonias escolares de vacaciones. Revista de Historia Contemporánea, 2, 94-124; Domènech, J. (2003). Las redes de centros educativos y la renovación pedagógica. La experiencia de la Coordinación de Escuelas 3-12 en Cataluña. Tabanque: Revista pedagógica, 17, 99-110; Pozo Andrés, Ma. Del M. (2004). La escuela nueva en España: crónica y semblanza de un mito. Historia de la educación: Revista interuniversitaria, 22-23, 317-346; Dávila Balsera, P. (2005). La renovación pedagógica en el País Vasco (segunda mitad del Siglo XX). Sarmiento: Anuario Gallego de Historia de la Educación, 9, 85-104; Roig López, O. (2006). La Escuela Moderna y la renovación pedagógica en Cataluña. Germinal: revista de estudios libertarios, 1, 75-84; Soler, J. (2009). La renovació pedagògica durant el Segle XX. La cruïlla catalana: dinamismes i tensions. Tesis doctoral. Universidad de Barcelona; y Groves, T. (2009). El Movimiento de Enseñantes durante el Tardofranquismo y la Transición a la Democracia 1970-1983. Tesis doctoral. UNED.

${ }^{10}$ Viñes, C., op. cit., 1983, 104. 
2. Corriente de carácter confesional, con una personalidad destacada, Andrés Manjón y las "Escuelas del Ave-María".

3. Corriente de carácter obrero, con la figura promotora de Ferrer Guardia y la "Escuela Moderna".

La "Institución Libre de Enseñanza" (en adelante ILE) fue una institución privada creada en 1876 por un grupo de Catedráticos, entre los que se encontraba Francisco Giner de los Ríos, Gumersindo de Azcárate, Teodoro Sainz Rueda, Nicolás Salmerón y otras personalidades profundamente comprometidas con la renovación pedagógica española. Separados de la Universidad Central de Madrid por defender la libertad de cátedra y negarse a amoldar sus enseñanzas a los dogmas oficiales en materia religiosa, política o moral, y fuertemente inspirados por el Krausismo de Karl Christian Friedrich Krause (debido a los viajes a Alemania de Julián Sanz del Rio que lo pusieron en contacto con sus ideas), desarrollaron un centro privado y laico ${ }^{11}$ que, en un primer momento comenzó dando enseñanza universitaria, y más tarde, se extendió a secundaria y primaria. Hasta la Guerra civil de 1936 la ILE tuvo un papel fundamental en la innovación cultural e intelectual, introduciendo en España las nuevas ideas y teorías pedagógicas que se estaban desarrollando en Europa. Se defendieron unas constantes pedagógicas que quedan caracterizadas a través de los siguientes puntos: desaparición de la enseñanza puramente memorística, participación de los alumnos, salidas al campo, relación de confianza entre docentes y alumnos, máximo respeto al niño, clima de tolerancia y relación con las familias. En definitiva, una educación activa, integral, armónica, gradual, co-educativa y laica.

La primera de las "escuelas del Ave María" de Andrés Manjón se creó en 1889 en Granada, pronto se extendió la iniciativa por el resto de España y a otros países, así, en 1896 existían 12 escuelas, ${ }^{12}$ llegando en 1920 a ser $300^{13}$. Dirigida a las capas más humildes de la sociedad, se daba una educación primaria gratuita donde la enseñanza de la religión católica era el centro de su pedagogía ${ }^{14}$. Los niños podían acceder a la edad de 3 años y se procuraba que, cuando dejasen la escuela, tuvieran una ocupación en la sociedad. Manjón mantuvo una posición crítica con la pedagogía tradicional, fomentando en sus escuelas metodologías nuevas y activas: enseñanza al aire libre, contacto con la naturaleza, juego, trabajo manual, enseñanza integral, orgánica y armónica, construcción del material escolar por los propios alumnos, enseñanza

\footnotetext{
11 "La Institución Libre de Enseñanza es completamente ajena a todo espíritu e interés de comunión religiosa, escuela filosófica o partido político; proclamando tan solo el principio de la libertad e inviolabilidad de la ciencia, y de la consiguiente independencia de su indagación y exposición respecto de cualquiera otra autoridad que la de la propia conciencia del profesor, único responsable de sus doctrinas". Estatutos de la ILE, artículo 15 (1877). Boletín de la Institución Libre de Enseñanza, 1, página de cabecera.

${ }^{12}$ Sin autor (1896, 5 de Enero). La lectura dominical, 105, 9.

${ }^{13}$ Canes, F. (1999). Las Escuelas del Ave María: una institución renovadora de finales del siglo XIX en España. Revista Complutense de Educación, 10 (2), 156.

${ }^{14}$ Manjón, A. (1947). Lo que son las escuelas del Ave-María. Madrid: Patronato de las Escuelas del AveMaría.
} 
artística... Tal como señalaba Aguilera en $1898^{15}$ : "en pocas escuelas de España se aplicarán de manera más prudente y eficaz los métodos y procedimientos de la pedagogía moderna como en las escuelas de D. Andrés Manjón".

Por último, la "Escuela Moderna" se crea en 1901 en Barcelona por Francisco Ferrer Guardia. Fuertemente influido por la ideología Anarquista y partiendo de la premisa de que la revolución pasa necesariamente por la educación, Ferrer crea un modelo racionalista de escuela, la "Escuela Moderna". Esta iniciativa fue modelo de otras que continuaron surgiendo por España y el extranjero. Propuso una enseñanza laica y racional: laica en contraposición a la escuela clerical, y racional y científica en favor de un desarrollo en libertad lejos de mitos y dogmas. Desde estos planteamientos pedagógicos se puso en práctica un ideario educativo profundamente renovador para la época: erradicación de los exámenes, las calificaciones y los castigos físicos; coeducación de sexos y de clases; y eliminación de todo conocimiento que no pudiese ser demostrado mediante el método científico ${ }^{16}$.

$\mathrm{Su}$ puesta en funcionamiento fue fruto de la donación económica de una alumna de Ferrer (Mademoiselle Meunier) que le apoyó en su proyecto. La "Escuela Moderna" no recibió ninguna ayuda económica pública, por lo que sólo se sustentó de las aportaciones que los padres depositaban de acuerdo a sus posibilidades. Esta iniciativa fue detenida en 1906 tras el atentado perpetrado por Mateo Morral (bibliotecario de la escuela) a Alfonso XIII. Tres años más tarde Ferrer fue fusilado acusado de instigar los sucesos de la "Semana Trágica de Barcelona" de 1909.

Debido al cierre de la "Escuela Moderna" de Barcelona y a la mala reputación ${ }^{17}$ que había despertado su modelo en algunos sectores de la población, desde la administración paulatinamente se fueron cerrando escuelas de carácter racionalista o negándose la autorización de apertura a las que estaban por abrir ${ }^{18}$.

\section{Del primer tercio del Siglo XX a la Guerra Civil (1936-1939)}

La crisis de 1998 sirvió de revulsivo definitivo para que los intelectuales españoles del ambiente regeneracionista y de renovación educativa buscasen decididamente la edificación de un país nuevo, una nueva sociedad, y por tanto, una nueva escuela. Comienzan a llegar a España, a través de diferentes órganos de expresión, las ideas y

\footnotetext{
${ }^{15}$ Aguilera, J. (1898). Don Andrés Manjón. Revista La Escuela Moderna, 93, 414.

${ }^{16}$ Ferrer, F. (1976). La Escuela Moderna. Barcelona: Tusquets.

17 "No es ésta ocasión oportuna para historiar con pormenores la vida de tales escuelas, la de Barcelona especialmente, de la cual hablan muchos sin conocerla, atribuyéndole algunos nada menos que la educación de todos los muchachos incendiarios de los conventos é iglesias barcelonesas en julio de 1909.” LLarena, J. (1919). Boceto de información mundial pedagógica. Revista La Escuela Moderna, 230, 779.

18 Este entorpecimiento o negación de apertura se aprecia en: Colominas, J. (1908). La enseñanza racionalista en España, pp. 270-274, en VV.AA (1978). Boletín de la Escuela Moderna. Edición de Albert Mayol, Barcelona: Tusquets; y en Sanmartí, T. (1907). Nuestra escuela, Revista Pedagógica Ilustrada (Órgano de la Escuela Moderna de Badalona, sostenida por la Sociedad protectora de la Enseñanza Racionalista), 6, 1.
} 
experiencias que estaban surgiendo dentro del movimiento de la Escuela Nueva en Europa $^{19}$. Así, surgen instituciones emblemáticas (herederas de los principios de la ILE) como la Junta para Ampliación de Estudios (en adelante JAE), que desde su creación en 1907 y hasta su disolución en 1939, pretendió acabar con el aislamiento científico, educativo y cultural que acontecía, desarrollando y posibilitando la modernización pedagógica de España. ${ }^{20}$.

A comienzos del Siglo XX en Cataluña, amplios sectores de la burguesía ligada al movimiento nacionalista comienzan a preocuparse por la renovación de la escuela y la promoción de la lengua y cultura catalana. Se cuestiona el modelo tradicional de escuela y se comienza a tomar contacto con las innovadoras ideas pedagógicas que llegaban de Europa, adscribiéndose a los principios de la Escuela Nueva. En líneas generales, esta búsqueda de una nueva realidad pedagógica se caracterizaba por varios elementos: acercamiento maestro-alumno, trabajo cooperativo, enseñanza del catalán, inserción de la escuela en el medio, descentralización y autonomía, gestión democrática, laicismo y coeducación ${ }^{21}$.

Esta preocupación logró cristalizar en la creación de varias escuelas privadas de carácter experimental, así, durante el primer tercio del Siglo XX, florecieron una amplia creación de centros que se propusieron renovar las prácticas escolares y modernizar Cataluña. Por tanto, tras la constatación de las deficiencias del sistema educativo vigente, gracias a la creación de la Mancomunitat surgen diferentes escuelas promovidas por la burguesía, fuertemente influenciadas por el movimiento cultural de la Renaixenca Catalana y el ideario pedagógico de la Escuela Nueva. Algunas de las más emblemáticas (ligadas a nombres como Rosa Sensat, Joan Bardina, Alexandre Galí, Pau Vila, Joan Palau Vera, Manuel Ainaud, Pere Vergés, Artur Martorell y Jeroni Estrany) se encuentran en las siguientes experiencias: colegio "Sant Jordi" (1898), escuelas "Mosén Cinto" (1904), colegio "Mont d Or" (1905), "Escuela Horaciana" (1905), "Granja Escolar Catalana" (1905), "Escoles Catalanes del Districte VI" (1906), "Vallparadis" (1910), escuela de la "Sagrada Familia" (1910), "Nuevo Colegio de Mont d’Or" (1913), "Escola del Bosc" de Monjuïc (1914), escuela racionalista

19 Sirva de ejemplo: Cousinet, R. (1924). La nueva educación. Boletín de la Institución Libre de Enseñanza, tomo XLVIII, 769, 97-99; Luzuriaga, L. (1923b). La educación nueva. Revista de Pedagogía, año II, 22, 361-367; y Vidal, J. (1924). La doctrina de la "nueva educación". Revista La Escuela Moderna, 7, 521-531.

${ }^{20}$ La JAE fue una institución predecesora del Consejo Superior de Investigaciones Científicas (CSIC) que contribuyó profundamente a la regeneración pedagógica de España, gracias a la creación de diferentes centros de investigación y un fructífero programa de becas o pensionados para estudiar en el extranjero. Algunas de las experiencias internacionales visitadas por los pensionados en Pedagogía fueron: las Escuelas Normales francesas Saint-Cloud, las escuelas-taller de Bélgica, el Instituto J. J. Rousseau y la Sorbona. Marín, T. (1989). La Renovación Pedagógica en España (1907-1936). Los pensionados en Pedagogía por la Junta para la Ampliación de Estudios. Madrid: Consejo Superior de Investigaciones Científicas.

${ }^{21}$ Caivano, F. y Carbonell, J., op. cit. 1979, s.p. 
“Galileo" (1915), “Casa del Bambini” (1915), "Escola del Mar" (1922), "Mutua escolar Blanquerna" (1923) y grupo escolar "Milá y Fontanals" $(1931)^{22}$.

En Madrid destaca la creación de las "escuelas de ensayo y reforma" que se implementaron en algunas escuelas graduadas ${ }^{23}$, concretamente las siguientes: "Grupo escolar Cervantes" (1918), "Príncipe de Asturias" (1918) e "Instituto-Escuela" (1918). Estas escuelas fueron centros experimentales financiados por los poderes públicos en los que se aplicaron las innovaciones metodológicas de la Escuela Nueva, esperando que fuesen posteriormente generalizadas al resto de escuelas públicas una vez demostrada su idoneidad ${ }^{24}$. Se lograron a través de los "Patronatos escolares", organismos legales y mediadores entre el Ministerio y las escuelas que posibilitaron la libertad necesaria a los centros (el primero creado en Madrid fue el Patronato de la Reina Victoria Eugenia en 1913).

Resaltando su situación de capital, de acuerdo a la inclinación centralista de las políticas del Gobierno, se pretendía situar a Madrid como germen y modelo de la renovación pedagógica española, el epicentro desde el cual se difundieran las ideas innovadoras al resto del país. ${ }^{25}$ Estos centros disfrutaron de un régimen de autonomía elevado a la hora de seleccionar a los maestros y de emprender experimentos al margen de las normativas del Ministerio. Asimismo, permitieron introducir nuevos contenidos (educación física, artística...) e implementar los métodos activos de la Escuela Nueva. Por tanto, se convirtieron en los principales iconos del regeneracionismo Español,

${ }^{22}$ En diferentes escuelas se ensayó específicamente el método Montessori como la "Casa del Bambini" (1915), el Colegio "Mont d'Or" (1905) o la escuela de la "Sagrada Familia" (1910). Payá, A. (2006). La actividad lúdica en la historia de la educación española contemporánea. Tesis Doctoral, Universidad de Valencia. Montessori puso en práctica un método que se basaba en el respeto hacia el niño, en su capacidad de aprender, en sus inclinaciones naturales y en las diferentes características propias de cada edad. Así, desarrolló ambientes educativos preparados con materiales didácticos específicos, libertad de movimientos, posibilidad de elección del trabajo deseado... La idea central era alcanzar el mayor grado de autonomía física y mental en los niños. Montessori, M. (1948). Ideas generales sobre mi método. Buenos Aires: Losada.

A nivel internacional el interés por el método Montessori cristalizó en la creación de diferentes ramas nacionales de la Asociación Montessori Internacional (Alemania, Austria, Suecia, Lituania, Rumania, Irlanda, Australia, Chile, EE.UU...) y en una multitud de escuelas que siguieron este método: Bulgaria (escuela experimental fundada por el profesor katzaroff), Colombia (escuelas públicas del distrito de Magdalena), Holanda (Liceo de Amsterdan)... Sin autor (1935). La labor del movimiento Montessori en diferentes países. Revista mensual ilustrada Montessori, año I, 5, 11-13.

${ }^{23}$ En 1899 con un Real Decreto del 23 de Septiembre se intenta introducir por vía de ensayo un modelo organizativo innovador que sólo se aplicaba en las escuelas de prácticas anejas a las normales, la escuela graduada. Viñao, A. (1990). Innovación pedagógica y racionalidad científica. La escuela graduada pública en España (1898-1936). Madrid: Akal. Tal como señalaba Ángel LLorca en 1922: "las más de nuestras escuelas son unitarias: un montón de niños de muy distintas edades y grado de cultura en una sola sala y a cargo de un solo maestro. Lentamente, con excesiva lentitud, las escuelas unitarias se convierten en graduadas: grupos homogéneos y relativamente reducidos de niños, una sala para cada grupo, en cada sala un maestro, y para todas las salas que constituyen la escuela, un director". LLorca, A. (1922) La escuela graduada en España. Revista de Pedagogía, año I, 7, 254.

${ }^{24}$ Pozo Andrés, Mª Del M., op. cit. 2004, pp. 317-346.

${ }^{25}$ Pozo Andrés, M ${ }^{\text {a }}$ Del M. (1996). La escuela graduada madrileña en el primer tercio del Siglo XX: ¿un modelo pedagógico para el resto del estado español? Revista Complutense de Educación, 7(2), 211-248. 
conformándose pronto en escuelas-modelo, objeto de numerosas visitas por parte de maestros y directores procedentes de todo el país. En 1934 el Gobierno de la CEDA suprime la autonomía y el carácter experimental de estas iniciativas, ${ }^{26}$ debido, entre otras cuestiones, a las reticencias que en los colectivos magisteriales habían suscitado estas experiencias, que no seguían los criterios de selección de profesorado por antigüedad, y que cuestionaban con su práctica la pedagogía tradicional.

El "Instituto-Escuela" de Madrid merece un análisis más detenido debido a su trascendencia y repercusión pedagógica en otras experiencias. Esta iniciativa se crea el 10 de Mayo de 1918 y finaliza con la Guerra Civil Española. Nace en una coyuntura histórica crítica, a nivel internacional, la Revolución Rusa de 1917 y la finalización de la I Guerra Mundial; y a nivel nacional, la Huelga General de Agosto de 1917, un Gobierno bipartidista que no funcionaba y una Monarquía de Alfonso XIII desgastada -peligrando el sistema de Restauración Borbónica iniciado tras la I República en 1874-. Así, entre 1917 y hasta el Golpe de Estado de Primo de Rivera en 1923, se intentó entre liberales y conservadores alcanzar algo de equilibrio creando gobiernos mixtos, durante el primer gobierno de ellos se crearía el Instituto-Escuela de Madrid.

Esta experiencia, influenciada pedagógicamente por la trayectoria de la ILE, abarcaba desde párvulos hasta la universidad y estaba sufragada por los fondos del Estado. Disfrutó de una tardía pero amplia repercusión, llegándose a crear otros durante la II República como el Instituto-Escuela de Barcelona (1931) ${ }^{27}$, Valencia (1932) y Sevilla $(1932)^{28}$. Fue un ensayo pedagógico creado por la JAE y dirigido a los hijos de la burguesía más ilustrada de Madrid $^{29}$ que también funcionó como una auténtica escuela normal donde se formaba pedagógicamente a las nuevas generaciones de docentes. Disfrutó de un amplio respaldo oficial y una considerable autonomía pedagógica, constituyéndose como una plataforma para la experimentación de diferentes innovaciones en el ámbito educativo ${ }^{30}$ : enseñanza activa, supresión de los

\footnotetext{
${ }^{26}$ Pozo Andrés, Mª Del M., op. cit. 2004, pp. 317-346.

${ }^{27}$ Fue una de las concesiones que obtuvo la Generalitat del Gobierno Republicano, el centro se sumó a la renovación pedagógica que se inició en Cataluña a principios del Siglo XX con la Escuela Moderna de Ferrer Guardia. Se renovó completamente el bachillerato Catalán, tal como señala una antigua alumna, prueba de ello se reflejaba en los métodos pedagógicos que se utilizaban: "no había exámenes ni libros de texto, para cada asignatura teníamos una libreta y a finales de la semana las revisaba el profesor". Tobarra, S. y Casals, D. (2007, 21 de Marzo). Reportaje. Un instituto pionero: sin libros, castigos ni notas, El Pais, s.p. http://elpais.com/diario/2007/03/21/catalunya/1174442855_850215.html (Consultado el 5 de Junio de 2012).

${ }^{28}$ Viñao, A. (2007). Reformas e innovaciones educativas en la España del primer tercio del siglo XX. La JAE como pretexto. Revista de educación, $n^{\circ}$ Extraordinario, ejemplar dedicado a Reformas $e$ innovaciones educativas (España, 1907-1939), 21-44.

${ }^{29}$ Fernández, R. y González, F. (2007). La Junta para Ampliación de Estudios e Investigaciones Científicas en el Centenario de su creación. Revista Complutense de Educación, 18 (1), 9-34.

30 R. D. de 10 de mayo de 1918, artículo I': "un Instituto escuela de segunda enseñanza, con residencias anejas para todos o una parte de los alumnos, en el que se aplicarán nuevos métodos de educación y planes de estudios. Al mismo tiempo se ensayarán en dicho centro sistemas prácticos para la formación del personal docente, adaptables a nuestro país." Un ensayo pedagógico, nuevo Instituto-escuela $(1918,11$ de Mayo). Diario La Época, p. 3.
} 
exámenes finales por evaluaciones continuas, prácticas de laboratorio, estudio directo de la naturaleza, lecturas comentadas, relación de la escuela con el medio, fomento de la curiosidad y la motivación interna del alumno, diálogo entre profesor y alumno... En síntesis, los principios que inspiraron su pedagogía quedan resumidos en 3 puntos: fomentar la curiosidad basando en ello todo el proceso didáctico, evitar en lo posible aquello que provocara un interés artificioso e inadecuado en el alumno y lograr un esfuerzo de trabajo basado en una motivación interna ${ }^{31}$.

Muchas de las experiencias que se gestaron durante la II República como el "Instituto-Escuela" de Barcelona, Valencia y Sevilla, o que siguieron desarrollándose durante la época como el "Instituto-Escuela" de Madrid o la "Mutua Escolar Blanquerna" de Barcelona, finalizaron con la llegada de la Guerra Civil.

\section{La Dictadura (1939-1975)}

Desde finales de la Guerra Civil (1936-1939) hasta mediados de los años 60, no puede hablarse más que de una involución en el terreno de las ideas y las prácticas escolares. ${ }^{32}$ Aun así, pese al desfavorable marco político y pedagógico generado durante el régimen de Franco a toda iniciativa pedagógica que tuviera un ligero matiz innovador, no se extirpó por completo la aparición o permanencia de algunas iniciativas de renovación pedagógica. Esta se desarrolló a través de iniciativas privadas localizadas principalmente en Cataluña y Madrid, mayoritariamente motivadas y gestadas dentro de una clase burguesa y media disconforme con el modelo educativo que se daba en la época. Así, en Cataluña se dieron algunos ejemplos significativos en las siguientes escuelas: "San Gregorio" (1955), "Talitha" (1956), "Instituto Costa i Llobera" (1957-1958), "Thau" (1963) y "Ton i Guida" (1963). En las inquietudes y motivaciones pedagógicas de los jóvenes maestros de estas experiencias se encontraba buena parte del origen y el fundamento de la escuela de Maestros Rosa Sensat, creada de forma clandestina por Marta Mata en 1965. Esta Institución ejerció una influencia sobresaliente en la formación de maestros ligados a la renovación pedagógica e influyó en la posterior creación en Madrid de los "movimientos de renovación pedagógica": ${ }^{33}$ “Acción educativa" (1975) y Escuela Abierta" (1980).

Siguiendo en Cataluña, durante la última época de la Dictadura varias de estas escuelas privadas se agruparon en el Colectivo de Escuelas para la Escuela Pública Catalana (CEPEPC), cuyo origen se encontraba en la "Coordinación Escolar" de años precedentes. La función del CEPEPC radicaba en coordinar esfuerzos dirigidos a la

31 Ruiz, J. (2006). Las reformas históricas de la enseñanza secundaria en España. Encounters on Education, 7, $95-111$.

${ }^{32}$ Caivano, F. y Carbonell, J., op. cit., 1979, s.p.

33 Algunos de los movimientos de renovación pedagógica (MRPs) como el Movimiento Cooperativo de Escuela Popular (MCEP) y Rosa Sensat, inician su camino en los años sesenta, pero la mayoría se ponen en funcionamiento a mediados de los setenta. Rogero, J. (2010). Movimientos de renovación pedagógica y profesionalización docente. Revista Latinoamericana de Inclusión Educativa, 4(1), 141-166. http://www.rinace.net/rlei/numeros/vol4-num1/art7.pdf (Consultado el 25 de Mayo de 2011). 
renovación pedagógica, buscar una alternativa al modelo de escuela estatal (a través de un modelo de gestión democrático y de participación) y plantear, en el proceso de democratización de la educación y la sociedad, el paso a la red pública de sus escuelas $^{34}$. Una vez se consiguió que las escuelas del CEPEPC ingresaran en la red de escuelas públicas ${ }^{35}$ el organismo se auto-disolvió.

Un ejemplo prototipo de aquellas escuelas del CEPEPC se encuentra en el Instituto Escuela "Costa i Llobera" (1957). Vigente en la actualidad, es un centro público con tres etapas educativas (infantil, primaria y secundaria) que se mantuvo inicialmente como centro privado ${ }^{36}$ caracterizándose desde el principio por ser una escuela innovadora y ligada a los movimientos de renovación pedagógica de Cataluña. $\mathrm{Su}$ vocación de transformarse en un centro escolar público la llevó a formar parte del movimiento de escuelas "Coordinación Escolar" y posteriormente de CEPEPC.

En Madrid surgen también una serie de centros educativos (fuertemente influidos por el ideario pedagógico de la Escuela Nueva) que logran evadir la normativa autoritaria impuesta por el régimen ${ }^{37}$. Así, algunas de las iniciativas más emblemáticas que siguen funcionando en la actualidad, las encontramos en las siguientes escuelas: colegio "Decroly"(1927), colegio "Estudio" (1940), colegio "Estilo" (1959) y colegio "Base" (1962).

El colegio "Decroly" (1927) es un centro concertado fundado por Ladislao Palenzuela. Su estilo de enseñanza se caracteriza desde el principio por adoptar un sistema pedagógico que tiende a una enseñanza personalizada, el uso de los métodos activos de Ovidio Decroly ${ }^{38}$ y el trabajo en equipo ${ }^{39}$. El colegio "Estudio" (1940) continuó las ideas pedagógicas, métodos de trabajo y técnicas de la ILE y del

\footnotetext{
${ }^{34}$ Domènech, J., op. cit., 2003, pp. 99-110.

${ }^{35}$ Maite Ricart señala que según el manifiesto del Conseller de Educación de la Generalitat Juan Guitart, en Diciembre de 1987 podría finalizar la integración de todos los centros de este colectivo a la red pública Catalana, algo que con un coste aproximado de 113 millones de pesetas, supondría un incremento de 22.000 alumnos, 1.261 profesores y 1.050 aulas para la red pública de la Generalitat. Ricart, M. (1987, 14 de octubre). Periódico semanal de información educativa "Comunidad Escolar", 161, 9. http://www.doredin.mec.es/documentos/00820083007647.pdf (Consultado el 22 de Mayo de 2012).

36 "Pero después de 30 años de funcionamiento como centro privado, con la democracia se le planteó, como a muchos otros centros del movimiento de renovación pedagógica, la gran disyuntiva: integrarse en la red pública o continuar como centro privado". Guitart, J. (1999, 31 de Enero). Reportaje: Costa i Llobera: 40 años de renovación pedagógica, El País, hemeroteca, http://elpais.com/diario/1999/01/31/catalunya/917748452_850215.html (Consultado el 10 de Febrero de 2012).

${ }^{37}$ Groves, T., op. cit., 2009, 201.

${ }^{38}$ Principales innovaciones pedagógicas de Decroly: centros de interés (ejes organizados de aprendizaje que permiten el tratamiento de los contenidos en torno a un tema central, elegido de acuerdo a las necesidades e intereses de los alumnos) y principio de globalización (los niños aprehenden el mundo sobre la base de una visión de conjunto, es decir, el pensamiento del niño no es analítico sino sintético). Decroly, O. (2006). La función de globalización y la enseñanza y otros ensayos. Madrid: Biblioteca Nueva.

39 Principios del Colegio "Decroly" http://www.colegiodecroly.org/index.php?option=com content\&view $=$ article\&id=46\&Itemid $=55$ (Consultado el 01 de Junio de 2012).
} 
"Instituto-Escuela"40. Es un colegio privado que dio servicio a grupos reducidos de intelectuales de izquierda que permanecieron en Madrid. Su admisión quedaba reducida esencialmente a contactos y a la posibilidad de la familia de costear un precio elevado ${ }^{41}$. Surgió de la mano de antiguos profesores del "Instituto-Escuela" -algunos de ellos habían tenido relación con los laboratorios de la JAE o habían sido pensionados por la misma institución, otros tenían relación con el Centro de Estudios Históricos- motivados por la intención de construir, de acuerdo a la nueva y crítica coyuntura política propia de la posguerra, una institución que siguiese los ideales pedagógicos del "Instituto-Escuela" de Madrid desaparecido tras la Guerra Civil. El colegio "Estilo" (1959) es un centro privado fundado por Josefina Aldecoa e ideado para modernizar el modelo pedagógico de la Dictadura. Acogió en un primer momento a intelectuales, artistas y en general a todo el que pretendía una educación distinta para sus hijos, instaurando un modelo en sintonía con los valores del Krausismo y el modelo pedagógico de la ILE. Por último, el colegio "Base" (1962) es un centro privado que también pretende continuar el ideario pedagógico de la ILE, rechazando pautas tradicionales de autoritarismo, dogmatismo o de jerarquización de los alumnos en función de los resultados de aprendizaje. A nivel metodológico se propicia la aplicación de métodos activos, la colaboración, el sentido de la responsabilidad, el autocontrol, el ejercicio de la libertad responsable y la reflexión sobre el cuidado del medio ambiente ${ }^{42}$.

Durante los últimos años de la Dictadura siguieron surgiendo por toda la geografía española una serie de experiencias que, desde diferentes realidades, trataron de consolidar una alternativa de escolarización y de renovación pedagógica ${ }^{43}$. Así, en Madrid aparecen tres experiencias emblemáticas actualmente en funcionamiento: colegio público "Palomeras bajas" (1969), colegio privado-concertado "Siglo XXI" (1970) y colegio público "Trabenco" (1972).

El colegio público "Palomeras bajas" (1969) tiene su origen en un proyecto de escuela pública del año 1969 cuyos rasgos más generales se basaban en tres principios: investigación pedagógica orientada hacia la enseñanza individualizada, trabajo en equipo y relación directa con el barrio y con los padres para su progresiva participación en la vida escolar ${ }^{44}$. Tanto antes como ahora se ponen en práctica diferentes metodologías activas y participativas, fomentándose la adquisición de hábitos de autonomía y autogestión en los alumnos a través de asambleas y planes de trabajo. El colegio privado-concertado "Siglo XXI" (1970) fue creado por la Cooperativa COIS,

${ }^{40}$ Gallego, E. (2008). Centros escolares con patrimonio histórico. El Colegio "Estudio". Madrid. CEE Participación Educativa, 7, 126-132.

${ }^{41}$ Groves, T., op. cit., 2009, 202.

${ }_{2}^{4}$ Proyecto educativo del Colegio "Base", http://www.colegiobase.com/cbase/index.php?sectionid=105 (Consultado el 26 de Mayo de 2012).

${ }^{43}$ Ledesma, M. y Marrero (2001). Construyendo la democracia: el papel de las "alternativas pedagógicas", en VV.AA. (ed.). Desacuerdos. Sobre arte, políticas y esfera pública en el Estado Español, 6, 182-193. http://ayp.unia.es (Consultado el 20 de Enero de 2012).

44 Proyecto educativo Colegio Público Palomeras Bajas, http://www.palomerasbajas.org/Docs /ProyectoEducativo.pdf (Consultado el 15 de Julio de 2012). 
es una iniciativa que tuvo sus referentes pedagógicos en Freinet y la Escuela Nueva, cuyos objetivos principales fueron contribuir al desarrollo de la renovación pedagógica y la gestión democrática de la escuela. Desde el comienzo pretendió funcionar como un proyecto progresista, avanzado y abierto a las nuevas concepciones educativas y renovadoras. Algunas de las principales señas de identidad y principios educativos que orientan su modelo educativo son las siguientes: laico, democrático, vanguardista, humanista y abierto al medio ${ }^{45}$. Por último, el colegio público "Trabenco" es un centro promovido a través de un grupo de Cooperativistas en 1972, al llegar a un acuerdo con el Ministerio de Educación y Ciencia por el que la Cooperativa cedía sus locales y el Ministerio les ofrecía la posibilidad de seleccionar al profesorado, ${ }^{46}$ siempre y cuando fuese personal funcionario o interino. En "Trabenco" se pone en funcionamiento una metodología activa, democrática y dinámica ${ }^{47}$ tanto en la clase como en el centro (funcionamiento asambleario que permite la toma colectiva de decisiones, trabajo por proyectos, relación fluida con la comunidad abriendo la escuela al barrio, correspondencia escolar con otros centros...). ${ }^{48}$

La renovación pedagógica experimentada durante estos últimos años de la Dictadura en Valencia y País Vasco, va a estar estrechamente vinculada con la preocupación por introducir planteamientos metodológicos propios de la Escuela Nueva y la enseñanza y recuperación de la lengua valenciana y euskera respectivamente. Así, encontramos experiencias emblemáticas vigentes actualmente en

45 PEC (Proyecto Educativo del Centro) del Colegio Siglo XXI. Enero de 2012.. http://colegiosigloxxi.org/system/files/PEC\%20ColegioSigloXXI\%20Enero2012.pdf (Consultado el 10 de Julio de 2012).

${ }^{46}$ La mayoría del equipo docente se encontraba vinculado a diferentes movimientos de renovación pedagógica: M.C.E.P. (Movimiento Cooperativo Escuela Popular), Acción Educativa,...etc. Pumares, L. (2001) Estudio de los factores que posibilitan la continuidad de un proyecto curricular global en un medio social determinado: Trabenco. 25 años de innovación educativa. Universidad Complutense de Madrid. Facultad de Educación: Tesis Doctoral. www.ucm.es/BUCM/tesis/edu/ucm-t25298.pdf (Consultado el 10 de Abril de 2012).

${ }^{47}$ Propuesta pedagógica antiautoritaria y asamblearia que sigue las huellas de Freinet y algunos elementos parciales de A.S. Neill. Carbonell, J. (1979). Colegio Trabenco, Cuadernos de Pedagogía, 51. Freinet mantuvo una postura crítica con la escuela tradicional y los métodos que desarrollaba al considerar que no preparaba para la vida. Puso en práctica una pedagogía activa que partía de las necesidades e intereses del niño y articulaba el trabajo manual con el intelectual. La actividad escolar debía ser una tarea útil al individuo y al grupo. Algunas de las principales técnicas que implementó fueron: la correspondencia interescolar, la asamblea de clase, la clase-paseo, el texto libre y la imprenta escolar. Freinet, C. (1996). La escuela moderna francesa. Una pedagogía moderna de sentido común. Las invariantes pedagógicas. Madrid: Morata. Alexander Sutherland Neill fundó en Inglaterra en 1921 la escuela Summerhill. En líneas generales, algunos de los principios pedagógicos fundamentales que caracterizan el pensamiento de Neill y el funcionamiento de esta escuela (claro ejemplo de educación antiautoritaria) son: autogobierno, respeto y autorregulación como bases de la convivencia social; firme creencia en la bondad natural del niño y preparación para una vida equilibrada y feliz como finalidad última de la educación. Neill, A. S. (1976). Summerhill. Un punto de vista radical sobre la educación de los niños. Madrid: Fondo de Cultura Económica.

${ }^{48}$ Visita al centro realizada durante el curso 2010-2011. Agradezco desde aquí a E. Pacheco (maestra del CEIP Trabenco) con afecto, su invitación. 
las escuelas "La Tramuntana" (1968), "Las Carolinas" (1972) y "La Gavina" (1975) en Valencia ${ }^{49}$ y en la Ikastola "Langile" (1970) en País Vasco.

"La Tramuntana" (1968) fue una experiencia en la que se educaba con la pedagogía y las técnicas de Freinet ${ }^{51}$. Al poco tiempo de su creación las autoridades franquistas negaron su reconocimiento como centro privado, algo que se intentó solucionar, tanto transformando la escuela (que funcionaba como Sociedad Limitada) en una cooperativa de padres y madres como cambiando el nombre, pasó a llamarse "Mistral". Tras diferentes conflictos internos, en 1975 se disuelve la cooperativa de padres y madres y se transforma en una cooperativa de maestros, volviendo a cambiar el nombre de la escuela que pasa definitivamente a llamarse escuela "La Masía", actualmente funciona como centro privado concertado. En cuanto a la escuela concertada "Las Carolinas" (1972) decir que es una cooperativa de profesionales de la enseñanza y personal no docente donde se trabaja con programas innovadores de educación y que comprende las etapas de educación infantil, primaria y secundaria. En líneas generales su proyecto educativo se caracteriza por ser: no autoritario, científico, ético, activo, democrático, valenciano, ecologista y co-educativo ${ }^{52}$. Por su parte, la escuela "La Gavina" (1975) surge de la iniciativa de un grupo de profesionales de la enseñanza agrupados en cooperativa, es una escuela pionera en la enseñanza en valenciano que se define desde sus inicios como co-educadora, plural, laica y valenciana. Se utiliza una metodología activa en la que el alumnado participa en todo tipo de tareas organizativas y de aprendizaje, poniéndose un especial empeño en la cooperación entre familia y escuela, actualmente funciona como escuela concertada. Por último, la Ikastola "Langile" (1970) en País Vasco es una iniciativa que "comenzó con la aplicación de una metodología Freinetiana." ${ }^{, 53}$ Inicialmente abrió sus puertas con un parvulario, posteriormente en 1972 se unifican las aulas de preescolar y en 1976 se legaliza la experiencia como cooperativa. Finalmente en 1993 pasa a integrarse en la red pública de escuelas de la Comunidad Autónoma Vasca.

En Galicia durante el curso 1972-73, de la mano de Teresa Ubeira y Juan Rodríguez de Llauder como fundadores, surge la significativa experiencia de O Pelouro. Un

\footnotetext{
49 Otra experiencia Valenciana emblemática se encuentra en el colegio público "Castell de Almoines". Finalista de los prestigiosos premios "Marta Mata" en 2010.

${ }^{50}$ Desde la fundación en 1914 de la primera Ikastola, su relación con la renovación pedagógica necesita de un estudio más detenido, algo que excede las pretensiones de esta investigación. Un excelente y útil estudio se puede encontrar en Dávila Balsera., op. cit., 2005, pp. 85-104.

${ }^{51}$ La Tramuntana era una de "aquellas primeras escuelas alternativas a la escuela nacional oficial, donde enseñaban en valenciano, estaba en el campo, los niños y las niñas cultivaban el huerto, criaban conejos y gallinas, jugaban en el patio con taparrabos, no llevaban uniforme, hacían asambleas, investigaban el medio que les rodeaba, hacían textos libres, periódicos, tenían una imprenta, viajaban y se escribían con otras escuelas alternativas". Martínez Bonafé, J. (sin fecha). El ejemplo y la actualidad del sueño educativo republicano. Conferencia pronunciada en la Casa del Pueblo de Almansa, el 8 de Febrero de 2008. http://www.uv.es/bonafe/documents/AlmansaEl\%20ejemplo\%20y\%201a\%20ac.pdf (Consultado el 20 de Julio de 2012).

52 Principios educativos del colegio "Las carolinas". http://www.lescarolines.com/ (Consultado el 15 de Junio de 2012).

${ }^{53}$ Dávila Balsera., op. cit., 2005, pp. 85-104.
} 
centro de innovación psicopedagógica e integración de carácter concertado donde gran parte del alumnado requiere necesidades educativas especiales y que nace de la conjunción de los enfoques pedagógico y psiquiátrico (de los que proceden los fundadores). Esta escuela, nada convencional en el panorama educativo español, mantiene una postura crítica frente a un modelo de escuela rígida y competitiva, construyendo una cotidianidad escolar sin horarios, asignaturas, clases o actividades fijas $^{54}$.

\section{De 1975 al presente, una travesía en construcción}

Tras el periodo de transición democrática que se inicia con el fallecimiento de Franco en 1975, en 1978 se proclama la Constitución, concluyendo un largo periodo histórico de depuración, prohibición y entorpecimiento a cualquier idea o práctica pedagógica que fuera diferente a los ideales del régimen. Por tanto, creado un marco político de descentralización autonómica, desde distintas inquietudes y bajo diferentes procesos y dificultades, han ido proliferando una rica y plural cantidad de iniciativas educativas de todo tipo que constituyen una alternativa pedagógica en la actualidad. En coherencia con el objetivo de síntesis que ha guiado la investigación, se visibilizan únicamente ${ }^{55}$ tres experiencias emblemáticas: las "Comunidades de aprendizaje", la "escuela libre Micael” y el colegio público "Amara Berri”.

La primera "Comunidad de aprendizaje" surge en 1978 en la escuela de personas adultas "La Verneda-Sant Martí" de Barcelona. En la actualidad hay 116 centros, mayoritariamente de titularidad pública (entre infantil, primaria, secundaria y adultos) presentes por toda la geografía española. Las "Comunidades de aprendizaje" son el resultado de las investigaciones del "Centro de Investigación Social y Educativa" de la Universidad de Barcelona (CREA) y de la experiencia educativa de tres proyectos de éxito situados en Estados Unidos en el ámbito de la educación infantil y primaria: las "Escuelas intensivas o aceleradas" ("Accelerated Schools" de la Stanford University) y los programas "Desarrollo escolar" ("School development Program" de la Yale University) y "Éxito para todos" ("Success for all” de la Johns Hopkins University).

Una "Comunidad de aprendizaje" se define como un proyecto de transformación social y cultural de un centro educativo y de su entorno con el que se pretende conseguir una sociedad de la información para todas las personas. Todo ello basado en un aprendizaje dialógico y mediante una educación participativa de la comunidad que

\footnotetext{
54 “O Pelouro es una escuela que no parece lo que convencionalmente nos hemos acostumbrado a entender por tal. Ni por sus espacios, ni por el tipo de niños y niñas que conviven en ella, ni por la forma en que se relacionan entre ellos -independientemente de su edad y de sus características personales-, ni por el tipo de presencia y de intervención de los adultos - ya sean profesores u otros adultos que colaboran con la escuela o que trabajan en ella-, ni por el tipo de actividades que se desarrollan allí, ni por la forma en que transcurre el tiempo, ni por el ambiente que se respira". Contreras (2002). Vivir O Pelouro, Cuadernos de Pedagogía, 313, 49.

${ }^{55}$ Durante los últimos años han seguido apareciendo interesantes iniciativas como el proyecto Atlántida e infinidad de experiencias como la escuela "Els Donyets" (1993) en Valencia, el CEIP "La Navata" (1994) en Madrid, o la escuela "El Roure" (2001) en Cataluña por citar algunas. Experiencias que siguen reconstruyendo en la actualidad la historia de la renovación pedagógica.
} 
se concreta en todos sus espacios posibles, incluido el aula. ${ }^{56}$ Una de las características distintivas de estas escuelas es la implementación de los "grupos interactivos", a través de ellos se permite democratizar el aula y superar la segregación ${ }^{57}$, así como modificar su distribución espacial tradicional reforzando la participación de diferentes agentes educativos $^{58}$. En definitiva, la base que sustenta las "Comunidades de aprendizaje" es el aprendizaje dialógico (siendo el resultante de un diálogo igualitario, horizontal y democrático donde el poder se sitúa en los argumentos) y los principios pedagógicos que las orientan son la participación democrática, la centralidad del aprendizaje, las expectativas altas y positivas y el progreso permanente.

La "escuela Libre Micael" (1979) implantó en España por primera vez la pedagogía Waldorf $^{59}$ creada por Rudolf Steiner (metodología aplicada actualmente en más de 2000 centros educativos de carácter público y privado repartidos en más de 70 países). Esta pedagogía fue creada al calor del movimiento de la Escuela Nueva de finales del Siglo XIX y comienzos del Siglo XX, constituye una notable aportación a la renovación pedagógica avalada por una larga trayectoria histórica. La "escuela Libre Micael" abrió primeramente sus puertas para educación infantil y posteriormente, en 1987 para educación primaria. En la actualidad es un centro privado de educación infantil, primaria, secundaria y bachillerato que apuesta por la creatividad del maestro, la participación democrática, la educación integral, la colaboración escuela-familia y la innovación educativa, ${ }^{60}$ tratando de armonizar la educación escolar con la familiar.

Por último, el colegio público "Amara Berri” es una iniciativa cuya principal impulsora fue Loli Anaut. Se inicia en 1979 fruto de una experiencia previa frustrada en un centro privado de Durango en 1972, allí se gestaron las bases pedagógicas futuras. Este centro da nombre al sistema pedagógico que ha cristalizado en una amplia red de centros en País Vasco, concretamente 18 escuelas públicas y 1 escuela

\footnotetext{
${ }^{56}$ Definición obtenida de acuerdo a: Elboj, C., Puigdellívol, I., Soler, M., y Valls, R. (2002). Comunidades de aprendizaje. Transformar la educación. Barcelona: Grao; VV.AA. (2006). Transformando la escuela: Comunidades de aprendizaje. Barcelona: Grao; Ferrer, G. (2005). Hacia la excelencia educativa en las comunidades de aprendizaje: participación, interactividad y aprendizaje. Revista Educar, Departamento de Pedagogía Aplicada, Universidad Autónoma de Barcelona, 35, 61-70; y Botton, L. (2009). La sociología dialógica de las Comunidades de Aprendizaje. Rase, Revista de la asociación de Sociología de la Educación, 2 (3), 6-13.

57 Puigvert, L. y Santacruz, I. (2006) La transformación de centros educativos en comunidades de aprendizaje. Calidad para todas y todos. Revista de Educación, 339, 169-176.

58 Entrevista abierta realizada el 12 de Abril de 2011 a la Directora de la Escuela de Educación Infantil El Zofio, centro transformado en Comunidad de aprendizaje. Agradezco desde aquí su colaboración y amabilidad durante la visita al centro.

${ }^{59}$ Pedagogía cuya base se encuentra en los fundamentos centrales de la Antroposofía, corriente filosófica de Rudolf Steiner. En líneas generales la pedagogía Waldorf plantea la educación como un desarrollo hacia la autonomía del ser humano. El objetivo principal es respetar el proceso madurativo de cada alumno y fomentar sus capacidades intelectuales, su voluntad y su sensibilidad artística. Steiner, R. (1980). La estructuración de la enseñanza basada en el conocimiento del hombre. México D.F.: Editorial Waldorf.

${ }^{60}$ Malagón, A. (2012). La Pedagogía Waldorf: la Escuela Libre Micael. Ponencia impartida en el II Seminario Pedagógico "Los movimientos sociales, renovación pedagógica y prácticas de educación alternativa", Coordinado por la Profesora Doña Sara Ramos Zamora, celebrado el en la Facultad de Educación de la Universidad Complutense de Madrid los días 29 y 30 de Mayo de 2012.
} 
concertada $^{61}$. La investigación, la experimentación y la innovación han sido y son una característica del Sistema Amara Berri, un sistema donde el proyecto educativo y la organización se encuentran armónicamente articulados con la estructura y la vida cotidiana del centro ${ }^{62}$. Su programación no se realiza por materias o centrados en unidades didácticas, sino a través de actividades llenas de contenido lúdico, actividades vitales donde las materias se interrelacionan, es lo que denominan: contextos sociales, estables y complementarios ${ }^{63}$ (contextos que constituyen la estructura básica de cada ciclo). En el año 1990 se considera a Amara Berri como Centro de Innovación Educativa del Departamento de Educación del Gobierno Vasco, reconociéndole las tres funciones que llevaba desarrollando hasta el momento: investigación, renovación pedagógica y acciones relacionadas con la formación del profesorado de otros centros.

\section{Conclusiones}

Sintetizando lo expuesto, la siempre incompleta historia de la renovación pedagógica española, alberga un rico y diverso legado de iniciativas que conforman una crónica que discurre paralela al momento histórico, político y social. Así, desde finales del Siglo XIX y comienzo del XX hasta la Dictadura Franquista, se observa el surgimiento de un amplio número de escuelas de distinta índole, fuertemente influenciadas por el ideario pedagógico de la Escuela Nueva que progresivamente fue introduciéndose en España. Tras la Guerra Civil y la posterior implantación del régimen, se clausuran muchas de las iniciativas de renovación pedagógica de mayor relevancia que se habían creado (principalmente durante la II República), dando comienzo a un largo periodo de retroceso e involución. A pesar del adverso marco pedagógico generado durante el régimen a cualquier iniciativa contraria a los ideales educativos que sostenía el mismo, surgen una serie de escuelas, principalmente desarrolladas a través de la iniciativa privada y mayoritariamente localizadas en Cataluña y Madrid, que constituyen una alternativa de renovación pedagógica en la época. Posteriormente, con la llegada de la democracia y hasta la actualidad (ya en un marco político de descentralización autonómica) han seguido surgiendo un variado número de centros escolares públicos y privados por toda la geografía española, bajo un ideal común de renovación de las prácticas escolares.

Considerando las diferentes realidades históricas y geográficas donde se generaban las iniciativas y bajo las diferentes influencias que las motivaba, desde finales del Siglo

\footnotetext{
${ }^{61}$ E. Guerrero González y E. Martín González (docentes del Colegio Amara Berri, actualmente trabajando en labores de formación y asesoramiento pedagógico del sistema Amara Berri), comunicación personal durante visita al centro realizada el 24 de Mayo de 2012. Agradezco desde aquí su colaboración y amabilidad.

${ }^{62}$ Carbonell, J. (1995), Centro de innovación educativa "Amara Berri”. La globalización como proceso vital. Cuadernos de Pedagogía, 235, 28-35.

${ }^{63}$ E. Guerrero González y E. Martín González (2012). Red Sistema Amara Berri. Ponencia impartida en el II Seminario Pedagógico "Los movimientos sociales, renovación pedagógica y prácticas de educación alternativa", coordinado por la Profesora Doña Sara Ramos Zamora, celebrado en la Facultad de Educación de la Universidad Complutense de Madrid los días 29 y 30 de Mayo de 2012.
} 
XIX y hasta nuestros días, se pone de manifiesto la no interrupción en el surgimiento de una amplia y heterogénea cantidad de centros escolares que han experimentado e impulsado procesos de renovación pedagógica en España. Una historia que, pese a mantenerse constante en el tiempo, transcurre a través de etapas de considerable apogeo, como la experimentada durante la II República y a partir de los años 70, y mayor decadencia, como la padecida durante la Dictadura.

Los actuales debates que enfrenta la educación sobre cómo responder con acierto a los nuevos retos que se plantean en nuestras sociedades, demandan una formación pedagógica e histórica sólida que permita aumentar y enriquecer el marco de discusión. En este sentido, el conocimiento de la evolución de la renovación escolar en España, posibilita un imaginario pedagógico heterogéneo y un conocimiento histórico y educativo más formado. Este trabajo supone una contribución al estudio de la historia de la educación española $\mathrm{y}$, en definitiva, a una completa formación de los profesionales de la educación.

En conclusión, el recorrido realizado expone un marco general de la historia de la renovación pedagógica desarrollada en España, estudiada a través de algunas de las escuelas más emblemáticas del panorama pedagógico, permitiendo disponer de una valiosa información que esclarece su evolución, muestra los diferentes idearios pedagógicos de cada experiencia y genera una necesaria visión de conjunto entre el pasado y el presente.

\section{Referencias bibliográficas}

AGUILERA, J. (1898). Don Andrés Manjón. La escuela Moderna, año VIII, 93, 414.

BOTTON, L. (2009). La sociología dialógica de las Comunidades de Aprendizaje. Rase, Revista de la asociación de Sociología de la Educación, 2 (3), 6-13.

CAIVANO, F. y CARBONELL, J. (1979). La Renovación Pedagógica ayer y hoy. Cuadernos de Pedagogía, 59, s.p.

CANES, F. (1999). Las Escuelas del Ave María: una institución renovadora de finales del siglo XIX en España. Revista Complutense de Educación, 10 (2), 156.

CARBONELL, J. (1979). Colegio Trabenco. Cuadernos de Pedagogía, 51, 21-24.

CARBONELL, J. (1995), Centro de innovación educativa "Amara Berri". La globalización como proceso vital. Cuadernos de Pedagogía, 235, 28-35.

COLOMINAS, J. (1908). La enseñanza racionalista en España, en VV.AA (1978). Boletín de la Escuela Moderna, pp. 270-274. Barcelona: Tusquets

CONTRERAS, J. (2002). Vivir O Pelouro, Cuadernos de Pedagogía, 313, 49.

COUSINET, R. (1924). La nueva educación. Boletín de la Institución Libre de Enseñanza, tomo XLVIII, 769, 97-99. 
DÁVILA BALSERA, P. (2005). La renovación pedagógica en el País Vasco (segunda mitad del Siglo XX). Sarmiento: Anuario Gallego de Historia de la Educación, 9, 85-104.

DECROLY, O. (2006). La función de globalización y la enseñanza y otros ensayos. Madrid: Biblioteca Nueva.

DOMÉNECH, J. (1995). Aproximació a la renovació pedagógica. Temps d’Educació, $14,175-184$.

DOMÉNECH, J. (2003). Las redes de centros educativos y la renovación pedagógica. La experiencia de la Coordinación de Escuelas 3-12 en Cataluña. Tabanque: Revista pedagógica, 17, 99-110.

ELBOJ, C., PUIGDELLÍVOL, I., SOLER, M., y VALLS, R. (2002). Comunidades de aprendizaje. Transformar la educación. Barcelona: Grao.

FERNÁNDEZ, R. y GONZÁLEZ, F. (2007). La Junta para Ampliación de Estudios e Investigaciones Científicas en el Centenario de su creación. Revista Complutense de Educación, 18 (1), 9-34.

FERRER, F. (1976). La Escuela Moderna. Barcelona: Tusquets.

FERRER, G. (2005). Hacia la excelencia educativa en las comunidades de aprendizaje: participación, interactividad y aprendizaje. Revista Educar, 35, 61-70.

FRABBONI, F. (1998). El libro de la pedagogía y la didáctica: II.- Lugares y tiempos de la educación. Madrid: Editorial Popular.

FREINET, C. (1996). La escuela moderna francesa. Una pedagogía moderna de sentido común. Las invariantes pedagógicas. Madrid: Morata

GALLEGO, E. (2008). Centros escolares con patrimonio histórico. El colegio "Estudio". Madrid. CEE Participación Educativa, 7,126-132.

GROVES, T. (2009). El Movimiento de Enseñantes durante el Tardofranquismo y la Transición a la Democracia 1970-1983. Tesis Doctoral. UNED.

ITE (Instituto Nacional de Tecnologías Educativas y de Formación del Profesorado). La renovación pedagógica en el Estado Español. http://www.ite.educacion.es/ (Consultado el 5 de Enero de 2012).

LEDESMA, M. y MARRERO, J. (2001). Construyendo la democracia: el papel de las "alternativas pedagógicas", en VV.AA. (ed.). Desacuerdos. Sobre arte, políticas y esfera pública en el Estado Español, 6, 182-193. http:// ayp.unia.es (Consultado el 20 de Enero de 2012).

LLARENA, J. (1919). Boceto de información mundial pedagógica. La escuela moderna, año $X X, 230,779$.

LLORCA, A. (1922) La escuela graduada en España. Revista de Pedagogía, año I, 7, 254. 
LUZURIAGA, L. (1923a). Las escuelas nuevas. Boletín de la Institución Libre de Enseñanza, tomo XLVII, 765, 304-309.

LUZURIAGA, L. (1923b). La educación nueva. Revista de Pedagogía, 22, 361-367.

MANJÓN, A. (1947). Lo que son las escuelas del Ave-María. Madrid: Patronato de las Escuelas del Ave-María.

MARÍN, T. (1989). La Renovación Pedagógica en España (1907-1936). Los pensionados en Pedagogía por la Junta para la Ampliación de Estudios. Madrid: Consejo Superior de Investigaciones Científicas.

MARTÍNEZ BONAFÉ, J. (sin fecha) El ejemplo y la actualidad del sueño educativo republicano. Conferencia pronunciada en la Casa del Pueblo de Almansa, el 8 de Febrero de 2008. http://www.uv.es/bonafe/documents/AlmansaEl\%20ejemplo\%20y\%201a\%20ac.pdf (Consultado 20 de Julio de 2012).

MATEU, J. (2011). Circunstancias, retos y posibilidades de las escuelas alternativas. Organización y Gestión Educativa, 4, 17-20.

MONTESSORI, M. (1948). Ideas generales sobre mi método. Buenos Aires: Losada.

NEILL, A. S. (1976). Summerhill. Un punto de vista radical sobre la educación de los niños. Madrid: Fondo de Cultura Económica.

PAYÁ, A. (2006). La actividad lúdica en la historia de la educación española contemporánea. Tesis doctoral. Universidad de Valencia.

POZO ANDRÉS, M ${ }^{\mathrm{a}}$ Del M. (1996). La escuela graduada madrileña en el primer tercio del Siglo XX: ¿un modelo pedagógico para el resto del estado español? Revista Complutense de Educación, 7 (2), 211-248.

POZO ANDRÉS, Ma . Del M. (2004). La escuela nueva en España: crónica y semblanza de un mito. Historia de la educación: Revista interuniversitaria, 22-23, 317-346.

PUIGVERT, L. y SANTACRUZ, I. (2006) La transformación de centros educativos en comunidades de aprendizaje. Calidad para todas y todos. Revista de Educación, $339,169-176$.

PUMARES, L. (2001) Estudio de los factores que posibilitan la continuidad de un proyecto curricular global en un medio social determinado: Trabenco. 25 años de innovación educativa. Tesis doctoral. Universidad Complutense de Madrid. www.ucm.es/BUCM/tesis/edu/ucm-t25298.pdf (Consultado el 10 de Abril de 2012).

ROGERO, J. (2010). Movimientos de renovación pedagógica y profesionalización docente. Revista Latinoamericana de Inclusión Educativa, 4 (1), 141-166. http://www.rinace.net/rlei/numeros/vol4-num1/art7.pdf. (Consultado el 25 de Mayo de 2011). 
ROIG, O. (2006). La Escuela Moderna y la renovación pedagógica en Cataluña. Germinal: revista de estudios libertarios, 1, 75-84.

RUIZ, J. (2006). Las reformas históricas de la enseñanza secundaria en España. Encounters on Education, 7, 95-111.

SANMARTÍ, T. (1907). Nuestra escuela, Revista Pedagógica Ilustrada (Órgano de la Escuela Moderna de Badalona, sostenida por la Sociedad protectora de la Enseñanza Racionalista), 6, 1.

SIN AUTOR (1935). La labor del movimiento Montessori en diferentes países. Revista mensual ilustrada Montessori, 5, 11-13.

SOLER, J. (2009). La renovació pedagògica durant el Segle XX. La cruilla catalana: dinamismes i tensions. Tesis doctoral. Universidad de Barcelona.

STEINER, R. (1980). La estructuración de la enseñanza basada en el conocimiento del hombre. México D.F.: Editorial Waldorf

VIDAL, J. (1924). La doctrina de la "nueva educación". Revista La Escuela Moderna, 7, 521-531.

VIÑAO, A. (1990). Innovación pedagógica y racionalidad científica. La escuela graduada pública en España (1898-1936). Madrid: Akal.

VIÑAO, A. (2007). Reformas e innovaciones educativas en la España del primer tercio del siglo XX. La JAE como pretexto. Revista de educación, $n^{\circ}$ Extraordinario, ejemplar dedicado a reformas e innovaciones educativas (España, 1907-1939), 2144.

VIÑES, C. (1983). La Renovación Pedagógica del Siglo XIX y las colonias escolares de vacaciones. Revista de Historia Contemporánea, 2, 94-124.

VV.AA. (2006). Transformando la escuela: Comunidades de aprendizaje. Barcelona: Grao.

\section{Correspondencia con el autor:}

Francisco Javier PERICACHO GÓMEZ

Departamento de Teoría e Historia de la Educación

Facultad de Educación, Universidad Complutense de Madrid

c/ Rector Royo Villanova s/n

28040 Madrid, España

Tlf: 686205295

e-mail: franciscojavier.pericacho@pdi.ucm.es 